\title{
Performance Analysis of Coded OFDM Signal for Radio over Fiber Transmission
}

\author{
Shikha Mahajan ${ }^{1}$, Naresh Kumar ${ }^{2}$ \\ ${ }^{I}$ (ECE, U.I.E.T/ Panjab University, India) \\ ${ }^{2}$ (Assistant Professor, ECE, U.I.E.T/Panjab University, India)
}

\begin{abstract}
The main aim of this paper is to analyze the influence of the modulation techniques; on the performance of coded orthogonal frequency-division multiplexing (COFDM) based Radio over Fiber (RoF) system. The modelling of the COFDM scheme for RoF system has been completely done by using software Optisystem. Both analysis and simulation results are presented for 16-QAM and 16-QPSK modulation techniques and performance is analyzed for optical amplifier and Erribium Doped Fiber Amplifier (EDFA). The parameter Quality factor $(Q)$ of the COFDM signal transmitted through the fiber optic link is examined. From the analysis for Radio over Multimode Fiber with link length of $2 \mathrm{~km}$ (medium haul communication) optical amplifier gives better results than EDFA for either of the modulation schemes for set of bit rates.
\end{abstract}

Keywords - COFDM, Convolutional Encoder, Multimode Fiber, Quality Factor, Radio over Fiber

\section{INTRODUCTION}

RoF technology is one of the recent advancements in optical communication, finding applications in cable TV networks, base station links for mobile communication and antenna remoting [1]. Particularly, radio over multimode fiber system (ROMMF) has gained much attention recently for its suitability to deliver highpurity signals in analog and digital format for short-reach coverage such as wireless local area networks and ultrawideband radio signals [2].

COFDM has been specified for digital broadcasting systems for both Digital Audio Broadcasting (DAB), Digital Video Broadcasting (DVB-T) [3]. COFDM is particularly well matched to these applications, since it is very tolerant of the effects of multipath (provided a suitable guard interval is used). Indeed, it is not limited to 'natural' multipath as it can also be used in so-called Single-Frequency Networks (SFNs) in which all transmitters radiate the same signal on the same frequency. A receiver may thus receive signals from several transmitters, normally with different delays and thus forming a kind of 'unnatural' additional multipath. Provided the range of delays of the multipath (natural or 'unnatural') does not exceed the designed tolerance of the system (slightly greater than the guard interval) all the received-signal components contribute usefully. For short-distance applications, COFDM can be used efficiently for high data rate signal delivery and to tolerate the effect of modal dispersion. Also, the exponent refractive index profile effect has been studied and shown that the best performance can be achieved near the optimum value of exponent refractive index profile [4].

COFDM system employs conventional forward error correction codes and interleaving for protection against burst errors caused by deep fades in the channel. Often it is concatenated with a block code for improving the performance [5]. A Viterbi decoder for decoding a convolution code is easy to implement. Codes of different rates are used in conjunction with different modulation schemes to support different quality of services. While error correction codes provide coding gain in the system, interleaving provides diversity gain. For COFDM, when a deep fade occurs in the channel the bits within the deep fade are erased. Interleaving the bits across different frequency bins distributes the energy within a symbol among different sub-carriers. Since distinct sub-carriers undergo different fading conditions, the probability that all the bits corresponding to a symbol are lost, decreases significantly. An uncoded OFDM system cannot exploit frequency diversity.

Modal dispersion is the dominant performance limiting factor in MMFs. The advantage of MMFs over single mode fibers (SMFs) is their relaxed coupling tolerance, their larger core diameters, which lead to reduced system-wide installation and maintenance costs. MMFs allow the propagation of multiple guided modes albeit with different propagation constants. The difference in the mode propagation times leads to intermodal dispersion, which severely limits the fiber bandwidth.

In ROF, COFDM impact has been demonstrated with MMF as a promising modulation scheme for mitigating the modal dispersion penalty [6]. By implementing coding and interleaving across sub-carriers, the 
effect of multipath fading is minimised since interleaving reorganises the number of bits in a way so as to avoid the effects of fading. Convolutional coding is being used commonly in COFDM systems, and it can give coding gain at different coding rates [7]. Thus, COFDM makes the transmitted signal robust to multipath fading and distortion. Also it has been observed in [8] that the frequency selectivity becomes significant with increasing the length of the MMF. This reveals the need for COFDM.

\section{SIMULATION SETUP FOR RADIO OVER FIBER SYSTEM}

RoF system is very cost effective because localization of signal processing in central station and use of a simple base station. It realizes transparent transformation between RF signal and optical signal. Fig 1 shows implementation approach for transmitting convolutionally encoded data over a multi mode fiber.

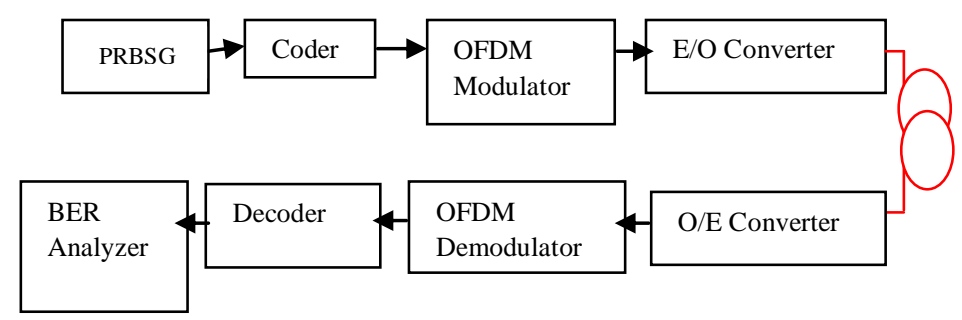

Fig. 1.Block Diagram of COFDM based RoF System

Pseudo-random bit sequence generator (PRBSG) data format is used which is convolutionally encoded at rate $1 / 2$ and generator polynomials $133_{8}$ and $171_{8}$. For downlink simulation, a narrow bandwidth continuous wave $(\mathrm{CW})$ (wavelength, $1300 \mathrm{~nm}$ ) from laser diode is modulated via a Mach-Zehnder Modulator (MZM). An ideal pre amplifier before optoelectronic conversion by PIN-PD having centre frequency of $1300 \mathrm{~nm}$ and post amplifier is used at uplink simulation. Optical link i.e. a multi mode fiber of length $2 \mathrm{~km}$ is used. In receiver section, optical signal is detected by a PIN-photodiode. Q-factor values of signals are measured by BER analyzer at base station.

\section{COFDM MODEL}

The implementation process of COFDM is as follows:

- The data bits are convolutionally encoded with a code rate $1 / 2$.

- The encoded bits are bit-interleaved and then mapped into in-phase and quadrature components of the complex symbol.

- The complex symbols are modulated by OFDM.

- At the receiver, direct detection is used. Table 1 summarises COFDM parameters.

- The decoding process employs the hard-decision viterbi decoding algorithm.

\begin{tabular}{ll}
\multicolumn{2}{c}{ Table 1.COFDM parameters } \\
\hline Parameter & Value \\
\hline Code rate & $1 / 2$ \\
Generator polynomials & $133_{8}, 171_{8}$ \\
Constraint length & 7 \\
\hline
\end{tabular}

\section{MMF RESPONSE MODEL}

The implementation process of MMF is as follows:

- COFDM signal generated out of the OFDM transmitter is injected into the laser diode (LD).

- The optical intensity signal out of the LD is fed into an MMF followed by a photodetector. 
- The received COFDM signal is demodulated and decoded to retrieve the data. Table 2 summarizes MMF parameters.

Table 2.MMF parameters

\begin{tabular}{ll}
\hline Parameter & Value \\
\hline Fiber dispersion & $-100 \mathrm{ps} /(\mathrm{nm}$ \\
& $\mathrm{km})$ \\
Fiber attenuation & $1.25 \mathrm{~dB} / \mathrm{km}$ \\
Wavelength & $1300 \mathrm{~nm}$ \\
Fiber length & $2 \mathrm{~km}$ \\
\hline
\end{tabular}

V. RESULTS AND DISCUSSION

In this section we have tried to investigate the impact of modulation techniques on RoF system. For RoF approach, multiple data signals are analyzed using optical and RF spectrum analyzers.

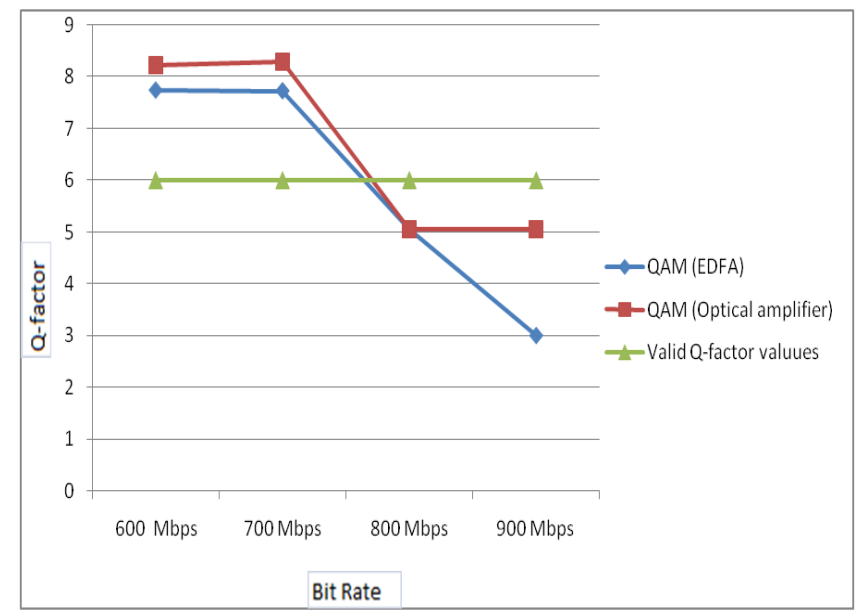

Fig. 2.Comparative analysis of QAM using optical amplifier and EDFA

From the Fig 2, above it can be seen that for bit rates used in simulation, QAM with optical amplifier gives better Quality factor than QAM with EDFA. Also as the bit rate increases the quality factor begins to degrade for both the modulation techniques for values greater than equal to $800 \mathrm{Mbps}$ and falls below the value required for communication purpose. The fall in the $\mathrm{Q}$ factor is due to increase in dispersion as bit rate increases. 


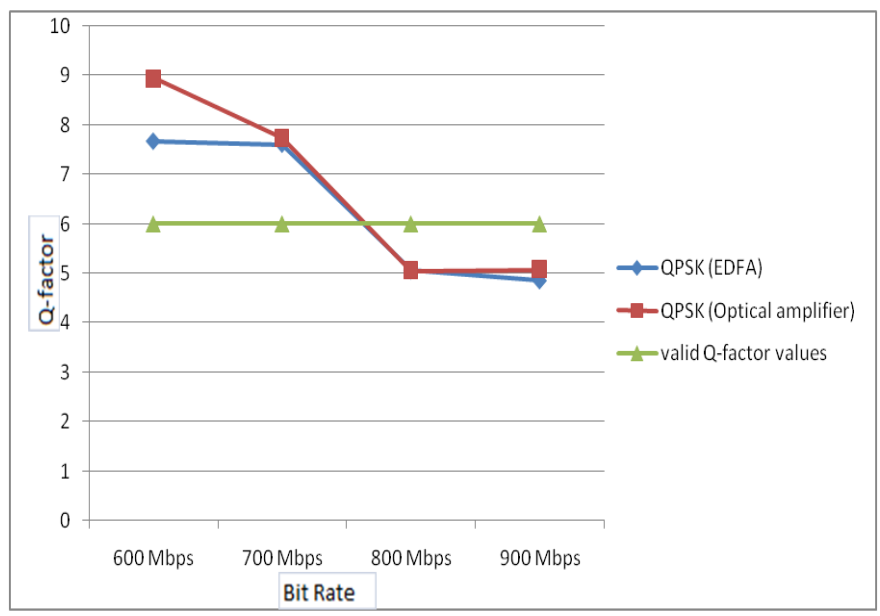

Fig. 3.Comparative analysis of QPSK using optical amplifier and EDFA

From the Fig 3, above it can be seen that for bit rates used in simulation, QPSK with optical amplifier gives better Quality factor values than QPSK with EDFA. Also as the bit rate increases the quality factor begins to degrade for both the modulation techniques for values greater than equal to $800 \mathrm{Mbps}$ and falls below the value required for communication purpose. The fall in the $\mathrm{Q}$ factor is due to increase inn dispersion as bit rate increases.

From above two figures 2 and 3 , it can be analyzed that either of the modulation techniques i.e. QAM or QPSK gives better performance with optical amplifier than used with EDFA.

\section{CONCLUSION}

From the results for Radio over MMF with link length of $2 \mathrm{~km}$ (medium haul communication) based upon the comparative analysis between QAM and QPSK modulation techniques with optical amplifier and EDFA for same values of increasing bit rates, optical amplifier gives better results than EDFA for either of the modulation techniques. After $800 \mathrm{Mbps}$ the quality factor value falls below permissible value for communication.

\section{REFERENCES}

[1] Hamed Al Raweshidy, Shozo Komaki, Radio over fiber technologies for mobile communications networks, pp. 128-132 (2002).

[2] Guennec, Y.1., Pizzinat, A., Meyer, S., et al., Low-cost transparent radio-over-fiber system for in- building distribution of UWB signals, pp. 2649-2657 (2009).

[3] Stott, J.H., The DVB terrestrial (DVB-T) specification and its implementation in a practical modem, pp. 255-260 (1996).

[4] A.M. Matarneh, S.S.A. Obayya, Bit-error ratio performance for radio over multimode fiber system using coded orthogonal frequency division multiplexing, pp. 151-157 (2011).

[5] Arun Agarwal, Kabita Agarwal, Design and Simulation of COFDM for High Speed Wireless Communication and Performance Analysis, pp. 22-28 (2011).

[6] Dixon, B.J., R.D., Iezekiel, S., Orthogonal frequency-division multiplexing in wireless communication systems with multimode fiber feeds, pp. 1404-1409 (2001).

[7] Prasad, R., OFDM for wireless communication systems, Artech House, (2004).

[8] Aser M. Matarneh, S. S. A. Obayya, I. D. Robertson, Coded orthogonal frequency division multiplexing transmission over graded index multimode fiber, (2007). 